\title{
Death due to acute fatty liver in pregnancy
}

Vidanapathirana $\mathrm{M}^{1 *}$, Hettiarachchi $\mathrm{HAM}^{2}$, Munasinghe $\mathrm{KR}^{2}$

${ }^{1}$ Department of Forensic Medicine, Faculty of Medical sciences, University of Sri Jayewardenepura, Department of Forensic Medicine, Faculty of Medicine, Galle *Corresponding author: Tel: 0094-718244641. E-mail address:

mudithavidana@yahoo.co.uk

MLJSL. Vol 1. No 2. Aug. pp 42 - 45

\begin{abstract}
Introduction: Acute fatty liver of pregnancy (AFLP) results due to disordered metabolism of fatty acids by mitochondria in the mother, caused by deficiency ofdehydrogenase enzyme. This is a rare instance of a foetus causing metabolic disease in the mother. The 'working diagnosis' of the clinicians was HELLP syndrome. The exact cause of death was made clear only after the postmortem examination.
\end{abstract}

Case report : 24 year old primi with 34 weeks of gestation admitted for blood pressure monitoring. She developed jaundice, high blood pressure with proteinurea, marginally elevated liver enzymes, low platelets which warranted termination of pregnancy. Despite the termination of pregnancy, she developed renal failure and hepatic encephalopathy and died on the eleventh day after delivery. Post mortem histopathology of liver revealed that the architecture was not altered and micro-vesicular fat infiltrations in centrilobular and mid zones. Hepatocytes were swollen but inflammation was not evident. Other tissues did not show any fat infiltration. HELLP syndrome was excluded due to absence of macro-vesicular fat infiltration in modest quantities throughout the liver lobules. There were no lobular disarray with hepatocyte dropout, reticulin collapse and portal tract inflammation to suggest viral hepatitis. There were no evidence of drug induced, toxic or viral induced micro-vesicular steatosis. Reye's syndrome is characterized by uniform micro-vesicular fatty change in the liver and other tissues such as skeletal muscles, kidney and heart.

Conclusion: Having excluded all other possibilities, the cause of death was ascertained as AFLP and this emphasizes the value of correct histopathological diagnosis.

Keywords: micro vesicular fatty change, pregnancy 


\section{Introduction:}

The Forensic Pathologist has an important role in ascertaining the causes of death in pregnancy. To achieve this, good autopsy with full histopathology and other necessary investigations are essential [1]. In Sri Lanka, most common causes of maternal mortality are postpartum hemorrhage 32\%, toxemia (PIH) 24\%, criminal abortion $10 \%$, sepsis $3 \%$, and other causes $31 \% 2$. Acute fatty liver of pregnancy (AFLP) is a rare condition affecting approximately one in 15,000 pregnancies [2].

The case under discussion was clinically diagnosed and managed as HELLP syndrome but following autopsy investigations, it was diagnosed as acute fatty liver in pregnancy.

\section{Case report}

Twenty four year old primi in 34th week of gestation (with uneventful 1st and 2nd trimesters) admitted to Teaching Hospital Mahamodara for blood pressure monitoring. She developed jaundice, high blood pressure with proteinurea, WBC 6,000 (lymphocytes 70\%), marginally elevated liver enzymes (SGPT higher than SGOT), low platelets $(89,000)$ which warranted termination of pregnancy by emergency caesarean section. The indication was pregnancy induced hypertension progressing to HELLP syndrome. Despite the termination of pregnancy, she deteriorated up to the stage of renal failure and hepatic encephalopathy. Liver biopsy was not done as her clotting profile was not in favor of invasive procedures. Three days after the delivery, she was transferred to Teaching Hospital Karapitiya (THK) for haemodialysis. At ICU of THK she was managed with blood products, intravenous fluid, haemodialysis and anti hypertensive drugs. Eleven days after delivery she died due to a cardiac arrest.

Post mortem histopathology of liver (photomicrograph-01) showed that the architecture was not altered and microvesicular fat infiltrations with central nuclei in centrilobular and mid zones. Hepatocytes were swollen but inflammation was not evident. Other tissues did not reveal any fat infiltration.

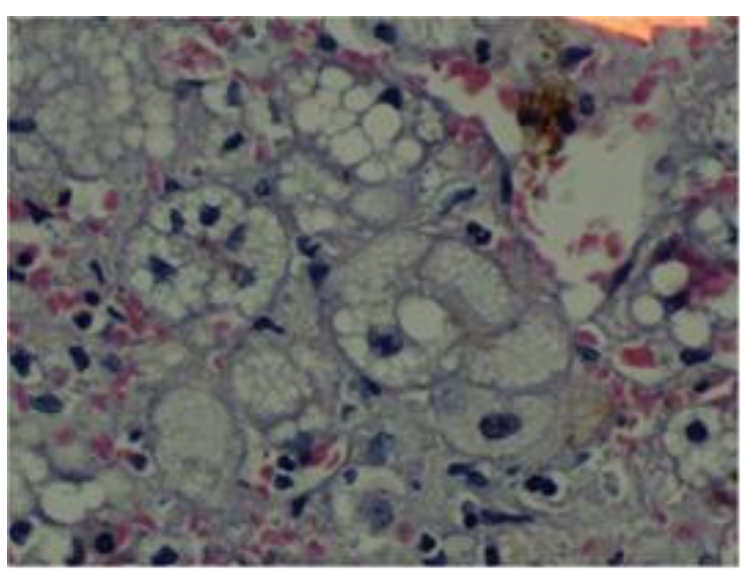

(Photomicrograph- $01 \mathrm{H} \& \mathrm{E})$ Micro-vesicular fat infiltration with central nuclei of mid and centrilobular hepatocytes.

\section{Discussion}

Acute fatty liver in pregnancy (AFLP) is caused due to disordered metabolism of fatty acids by mitochondria in the mother, caused by deficiency in a dehydrogenase enzyme with overload of fat from the fetus. It is a rare instance of a foetus causing metabolic disease in the mother [3]. Diagnosis of AFLP depends on, [1] A high index of suspicion [2] Confirmation of 
micro-vesicular steatosis and [3] Exclusion of other possibilities [4].

HELLP syndrome is a life-threatening obstetric complication usually considered to be a variant of pre-eclampsia. HELLP is an abbreviation of the main findings: Hemolytic anemia, elevated liver enzymes and low platelet count [5].

Features in favour of AFLP

AFLP is a disease of the third trimester whereas HELLP syndrome develops in the second trimester. Though AFLP can occur during any gestation, HELLP Syndrome affected women are less likely to be primiparous. AFLP can affect very young adults but HELLP Syndrome usually affects women over 25 years of age [6]. Most HELLP syndrome patients have hypertension and proteinurea which found in $50 \%$ of AFLP too ${ }^{5}$. SGPT is higher than the SGOT in AFLP, and in HELLP Syndrome it is the SGOT which is higher. Increased bilirubin level with jaundice is almost always seen in AFLP but it is not a feature of HELLP Syndrome. In latter stages of AFLP, prothrombin time increases, fibrinogen level and platelet count decreases due to DIC[6].

Despite similar settings and occasional clinical overlap, these two conditions are histologically distinct [7]. In AFLP the postmortem histopathology reveals microvesicular fat infiltration with central nuclei of centrilobular and mid zones, sparing a rim of peripheral hepatocytes as was found in this case. But in HELLP Syndrome macrovesicular fat is seen with eccentric nuclei distributed in modest quantities throughout the liver lobules[7]. Therefore, HELLP syndrome was excluded.
Viral hepatitis is another differential diagnosis for this clinical picture and was excluded due to absence of lobular disarray with hepatocyte dropout, reticulin collapse and portal tract inflammation [5].

Microvesicular steatosis with central nuclei is quite frequently observed in association with AFLP, certain types of medication eg. Tetracylines, Salicylates, valproate and in some metabolic disorders such as Reye's syndrome, and rarely seen in alocoholism[8].

Reye's syndrome affects primarily younger children and rarely adults. It is associated with certain strains of the influenza virus and often triggered by aspirin intake [9]. Clinically it presents with pernicious vomiting, convulsions, intractable hypoglycaemia and hepatomegaly [10]. Microvesicular steatosis with central nuclei is seen in liver and similar steatosis is found in skeletal muscles, renal tubular epithelium, and occasionally in the cardiac conduction system [9]. Though postmortem demonstration of viral antibodies was not done, Reye's syndrome was excluded due to the absence of such clinical features and fatty changes in other tissues such as muscle, kidney and heart.

There were no clinical evidence of drug induced, toxic or viral induced microvesicular steatosis.

In alcohol-related fatty liver, the vacuoles tend to be macrovesicular and displaces the nucleus eccentrically. Periportal area is relatively spared and Mallory bodies are characteristically present in alcoholic hepatitis. Only rarely microvesicular steatosis is the predominant pattern in alcohol-related fatty liver and is termed as alcoholic foamy degeneration [8]. She was 
not an alcoholic and was not on any longterm medications.

Finally the underlying cause of death was ascertained as AFLP. Most likely mechanism of death in fatty change of the liver is apparently an electrolyte imbalance with hypoglycaemia, hypokalaemia and fatty acid hyperlipidaemia. The mortality from AFLP has reduced significantly to $18 \%$, and is now related primarily to complications [5]. In this case, the terminal cardiac arrest could have been due to complications such as electrolyte imbalance and hypoglycaemia which may perhaps have been prevented.

\section{Reference}

1. Pekka S, Knight B., Knight's Forensic Pathology, 3rd Ed, Arnold, London, P. 431.

2. Family Health Bureau-Sri Lanka. Maternal mortality rate; 2005 [updated 2007; cited 2010 Dec 20] Available from: http://www.familyhealyh.gov.lk/anuradh apura.html.

3. Maitra A, Domiati SR, Yost $N$, et al. Absence of the G1528C (E474Q) mutation in the alpha sub unit of the mitochondrial tri functional protein in women with acute fatty liver of pregnancy. Pediatr Res. 2002;51:658-661.

4. Kumar V, Abbas AK, Fausto N. Robbins and Cotran Pathologic basis of disease. 7th edition. Philadelphia: Saunders; 2004.
5. Eugene R Schiff, Michael F Sorrel, Willis C Maddrey. Schiff's diseases of the liver.9th edition.2.Philadelphia: Lippincott Williams \& Wilkins; 2002.p.1439-1446.

6. Sibai B, Ramadan M, Chari $R$, et al. Pregnancies complicated by HELLP syndrome. Subsequent pregnancy outcome and long term prognosis. Am J Obstet Gynecol.1995; 172:125-129.

7. Halim A, Kanayama N, El Maradny E, et al. Immunohistochemical study in cases of HELLP syndrome and acute fatty liver of pregnancy. Gynecol Obste invest.1996; 41:106-112.

8. Uchida, T., Kao, H., Quispe-Sjogren, M., and Peters, R.L.,: Alcoholic foamy degeneration: a pattern of acute alcohol injury of the liver. Gastroenterology, 84(4):683-692, 1983.

9. Maheady, D.C.: Reye's syndrome: Review and update. J Paediatric Health Care, 3:246-250, 1989.

10. Meythaler JN, Varma RR. Ryes syndrome in adults. Diagnostic considerations. Ach Intern Med.1987; 147:61

\section{Contribution of authors}

Performing the autopsy- KRM, MH

Opinion- $\mathrm{MH}, \mathrm{KRM}, \mathrm{MV}$

Writing the manuscript $-K R M, M V$

Revising the manuscript- MV 